\title{
Effect of purification methods on the physicochemical and thermodynamic properties and crystallization kinetics of medium-chain, medium-long-chain, and long-chain diacylglycerols
}

\begin{abstract}
Medium-chain diacylglycerol (MCD), medium-long-chain diacylglycerol (MLCD), and longchain diacylglycerol (LCD) were prepared through enzymatic esterification using different conditions at temperatures of $55-70{ }^{\circ} \mathrm{C}$ and reaction times of $1.5-5 \mathrm{~h}$ and in the presence of 5-6\% Novozym 435. Subsequently, purification was performed using three different techniques, namely, molecular distillation (MD), deodorization (DO), and silica gel column chromatography (SGCC). Variations in terms of the physicochemical and thermodynamic properties, crystallization properties, and kinetics of the diacylglycerols (DAGs) before and after purification were determined. Irrespective of the DAG chain lengths, SGCC was able to produce samples with high DAG purity (96-99 wt \%), followed by MD (58-79 wt \%) and DO (39-59 wt \%). A higher 1,3DAG/1,2-DAG ratio was recorded for all samples, with the highest ratio recorded for SGCC purified samples. Regardless of the purification techniques used, the solid fat content (SFC) profiles of crude samples with steep curves were altered post-purification, showing a gradual increment in SFC along with increasing temperature. Modification of the Avrami constant and coefficient suggested the modification of the crystal growth mechanism post-purification. Crystallization and melting temperatures of products with a higher DAG purity were shifted to a higher temperature region. Variations were also reflected in terms of the crystal polymorphism, whereby the $\alpha$ and $\beta^{\prime}$ crystals transitioned into the more stable $\beta$ form in purified samples accompanied by modification in the microstructures and crystal sizes. However, there was insignificant change in the morphology of MLCD crystal after purification, except for the decrease in crystal sizes. In conclusion, synthesis of MCD, MLCD, and LCD comprising different DAG purities had distinctive SFC profiles, thermodynamic properties, crystallization kinetics, and crystal morphologies, which can be further incorporated into the preparation of a variety of fat products to obtain end products with desired characteristics.
\end{abstract}

Keyword: Enzymatic esterification; Diglycerides; Molecular distillation; Deodorization; Silica gel column chromatography; Acylglycerol composition; Structured lipid 